\title{
Studies on the external factors inducing the formation of turions in Spirodela polyrrhiza (L.) Schleiden
}

\author{
by
}

\section{CZOPEK}

\section{INTRODUCTION}

Spirodela polyrrhiza, a member of the Lemnaceae, is characterised by the ability to form turions, which differ from turions of other species of this family in respect of anatomy and morphology. Turions of this species are independent organs of about $1 / 7$ of the area of a vegetative frond, presenting an olive-green pigmentation and containing a great amount of storage substances (starch). Their water content is relatively high - about $61,8 \%$ on fresh weight basis ( $\mathrm{C} \mathrm{zopek} 1959 \mathrm{~b}$ ).

Turions of Spirodela polyrrhiza do not form any further turions, whereas turions of such species as Lemna minor and Wolffia have a certain ability to form them (L a ndolt 1957). After a period of dormancy the turions germinate and form fronds, the further multiplication of which proceeds on a vegetative way. An exact description of the anatomy and morphology of turions is given by $\mathrm{Jacobs}$ (1947).

Conditions leading to the formation of turions in a natural environment were examined by many authors. Hor en (1870, cit. acc. J a c o b s 1947) points out that turions are formed in stagnant waters exposed directly to solar radiations. Gup p y (1895, cit. acc. J a c o b s 1947) cbserved that turions are formed at relatively high temperature. A r ber (1920, cit. acc. J a c o b s 1947) suggests that conditions which influence favourably the vegetative growth should induce turions formation; sometimes however deficiency of nutrient solutions may cause the formation of these organs.

In a natural environment turions are formed from July till late autumn; in laboratory conditions, however, they may be obtained at any season of the year (Henssen 1954, C zopek $1959 \mathrm{~b}, 1962$ ). Investigations on the conditions favoring the formation of turions by fronds of Spirodela polyrrhiza were initiated by J a cobs (1947) who examined the influence of such factors as temperature, light intensity, photoperiodism, $\mathrm{CO}_{2}$ concentration and nitrogen deficiency. $\mathrm{H}$ ens sen 
(1954) examined the action of various sugars on the formation of turions and their enzymatic activity during dormancy and vegetative idevelopment. Czopek (1959 b) described the morphological differences in turions formed in light and darkness and insisted on the correlation between the turions formation and the dense crowding on the upper surface of the nutrient solution by vegetative fronds. H ill m a n (1961) described some factors influencing the formation of turions and their dormancy.

In spite of these investigations the problem of turions formation is far from being elucidated. For this reason a new study was undertaken on the action of several factors on the formation of turions. Its principal aim was to discover the factor (or factors) directly responsible for the formation of turions.

\section{MATERIAL AND METHODS}

Turions of Spirodela polyrrhiza formed by fronds grown in laboratory sterile cultures ( $\mathrm{Cz}$ opek $1959 \mathrm{a})$ provided the experimental material. They were stored in sterile conditions for about 1 month in complete darkness in a refrigerator (temperature $0-3^{\circ} \mathrm{C}$ ) and then allowed to germinate in $200 \mathrm{ml}$ Erlenmeyer flasks filled with $100 \mathrm{ml}$ Pirson and Seidel's nutrient solution with $1 \%$ sucrose (K a n deler 1955). After the formation of the first frond and the appearance of the initials of the second, the plants were transferred, under sterile conditions, into the proper culture vessels. In this way, a vegetative material of the same age was obtained for investigations on the formation of turions.

\section{Culture conditions}

The experiments were carried out on Pirson and Seidel's nutrient solution supplemented with $1 \%$ sucrose, its composition is given below: $\mathrm{KNO}_{3}-0,4 \mathrm{~g}, \mathrm{KH}_{2} \mathrm{PO}_{4}-0,2 \mathrm{~g}, \mathrm{MgSO}_{4} \cdot 7 \mathrm{H}_{2} \mathrm{O}-0,3 \mathrm{~g}, \mathrm{CaCl}_{2} \cdot 6 \mathrm{H}_{2} \mathrm{O}-0,61 \mathrm{~g}$, $\mathrm{MnCl}_{2} \cdot 4 \mathrm{H}_{2} \mathrm{O}-0,3 \mathrm{mg}, \mathrm{H}_{3} \mathrm{BO}_{3}-0,5 \mathrm{mg}$, ferric citrate $-5,0 \mathrm{mg}, \mathrm{H}_{2} \mathrm{O}$ dest. $-1000 \mathrm{ml}$ (K a ndele r 1955). $200 \mathrm{ml}$ Erlenmeyer flasks with $100 \mathrm{ml}$ nutrient solution (free area of the nutrient solution $-35 \mathrm{~cm}^{2}$ ) and smaller Erlenmeyer flasks and beaks were used for cultures. The nutrient solutions were autoclaved for 20 minutes under 1,5 Atm. pressure. The cultures were kept in a light thermostat in $28^{\circ} \mathrm{C}\left( \pm 1^{\circ} \mathrm{C}\right)$ in continuous white light emmitted by 6 fluorescent tubes $25 \mathrm{~W}$. The light intensity was $10660 \mathrm{ergs} / \mathrm{cm}^{2} / \mathrm{sec}$. 3 Erlenmeyer flasks were used in each series for each experimental combination and the corresponding means were calculated. For aeration of the nutrient solution air was pumped through suitable filters in order to assure complete sterility. 
A glass pipe ( $25 \mathrm{~cm}$ long, $4 \mathrm{~mm}$ in diameter) filled tight with cotton wool $(10 \mathrm{~cm})$ and a piece of filtration paper $(1 \mathrm{~cm})$ formed the filtration arrangement. The nutrient solutions were aerated daily for 10 minutes during the first 10 days and 30 minutes in the following ones.

Special transparent plexiglass rings were used in order to reduce the upper surface of the nutrient solution put at the disposal of the growing fronds withouth changing its total upper area. The rings were $4,5 \mathrm{~cm}$ high, the area of the bigger being $15,7 \mathrm{~cm}^{2}$ and that of the smaller

Fig. 1. Scheme of a culture vessel equipped with a ring and filter arrangement for aeration of the nutrient solution

1 - the inflow of air from the aeration arrangement; 2 - cotton wool cork; 3 - aperture for plant transferred; 4 -filter tube for aeration of the nutrient solution: 5 - ring; 6 - nutrient solution

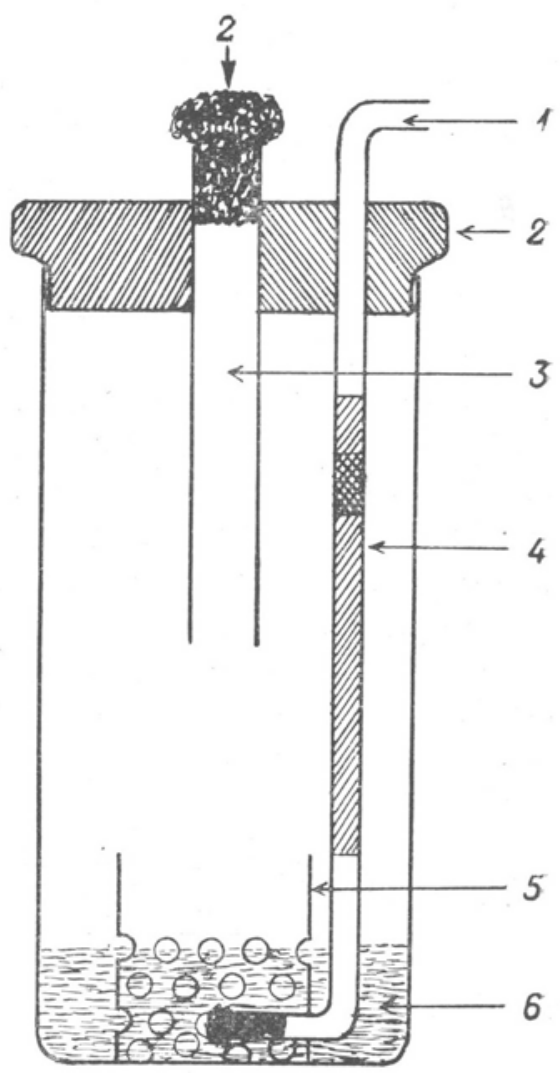

$9,5 \mathrm{~cm}^{2}$ (Fig. 1). The size of the side perforations (enabling the diffusion of the nutrient solution) was from $2,5 \mathrm{~mm}$ to $7 \mathrm{~mm}$ in diameter and their numbers were 81 and 56 in the bigger and smaller rings respectively. The rings were placed on the bottom of a beak of $54 \mathrm{~cm}^{2}$ area and the nutrient solution reached till the half of the height of the ring. The ring (through a side aperture) was connected with the filter tube through which sterile air was pumped (Fig. 1). 


\section{Computation of formed turions}

The ratio of the number of turions and the number of vegetative fronds in the Erlenmeyer flask was the criterion adopted for measuring the intensity of turions formation. Turions were usually counted after 7 weeks elapsing from the moment a sample was transferred with one plant. By this time, the process of turions formation has already come to an end. Moreover, the day the turions began to be formed was also recorded. The adopted criteria were 5 slightly visible turions initials and the percentage of the upper area of the culture solution covered by vegetative fronds.

The sucrose concentration in the solutions during the formation of turiens was determined by means of the Bertrand method.

\section{RESULTS}

1. Influence of the size of the upper surface of the nutrient solution

Previous investigations showed that turions are formed only when the whole surface of the nutrient solution has been covered with vegetative fronds ( $\mathrm{C} \mathrm{z}$ o p e k 1959b). This observation suggested that the size of the area put at the disposal of growing fronds may modify the length of time necessary for the formation of turions. An attempt was made to elucidate this problem by allowing Spirodela polyrrhiza fronds to grow in Erlenmeyer flasks in which the upper areas of the nutrient solutions were respectively 12, 35, 70 and $106 \mathrm{~cm}^{2}$. Each Erlenmeyer

Table 1

Number of vegetative fronds and turions in dependence on the upper free area of the nutrient . solution

\begin{tabular}{c|c|c|c|c}
\hline $\begin{array}{c}\text { Area of the nut- } \\
\text { rient solution } \mathrm{cm}^{2}\end{array}$ & $\begin{array}{c}\text { Number of ve- } \\
\text { getative fronds }\end{array}$ & $\begin{array}{c}\text { Number of tu- } \\
\text { rions }\end{array}$ & $\begin{array}{c}\text { Ratio of vegetati- } \\
\text { ve fronds to turions }\end{array}$ & $\begin{array}{c}\text { Day of turions } \\
\text { formation }\end{array}$ \\
\hline 12 & 153 & 984 & $1: 6.4$ & 18 \\
35 & 281 & 1143 & $1: 4.1$ & 23 \\
70 & 397 & 1434 & $1: 3.8$ & 24 \\
106 & 508 & 1783 & $1: 3.5$ & 27 \\
\end{tabular}

flask contained the same volume of the solution $(100 \mathrm{ml})$ and was provided with only one plant taken immediately after its germination from a turion and subsequently placed in a light thermostat. No aeration of nutrient solution was applied. After 7 weeks vegetative fronds and turions were counted (Table 1). 
It results from this experiment that on a small area turions are formed earlier than on a greater one; in the first case the whole area is sooner covered with vegetative fronds than in the second case. Owing to intense multiplication the fronds form several layers. Turions are formed first in the upper layers and then in the lower ones. The greater the free area of the nutrient solution the later the turions are formed. These experiments indicate that turions are rapidly formed in idense agglomeration of vegetative fronds.

\section{Reduction of the growth area in the nutrient solution}

The device used for reducing the area put at the disposal of growing fronds was described in the methodical part. Vegetative fronds cover the area inside the ring sooner than the area of the control cultures, but in both cases turions are formed at the same time. It results from

\section{Table 2}

Number of formed turions in dependence on the inside area of the ring and aeration

\begin{tabular}{c|c|c|c|c|c}
\hline $\begin{array}{c}\text { Total free area } \\
\text { solution } \mathrm{cm}^{2}\end{array}$ & $\begin{array}{c}\text { Area inside } \\
\text { the ring } \mathrm{cm}^{2}\end{array}$ & $\begin{array}{c}\text { Number of } \\
\text { vegetative } \\
\text { fronds }\end{array}$ & $\begin{array}{c}\text { Number of } \\
\text { turions }\end{array}$ & $\begin{array}{c}\text { Ratio of vegeta- } \\
\text { tive fronds to } \\
\text { turions }\end{array}$ & $\begin{array}{c}\text { Day of } \\
\text { turion } \\
\text { formation }\end{array}$ \\
\hline 54 & $\begin{array}{c}\text { without ring } \\
\text { (control) }\end{array}$ & 497 & 1173 & $1: 2.4$ & 22 \\
54 & $\begin{array}{c}15.7 \\
54\end{array}$ & 285 & 640 & $1: 2.2$ & 22 \\
54 & $\begin{array}{c}15.7 \\
\text { (aerated) } \\
9.5\end{array}$ & 363 & 711 & $1: 1.6$ & 21 \\
& $\begin{array}{c}9.5 \\
\text { (aerated) }\end{array}$ & 277 & 798 & $1: 2.0$ & 22 \\
\end{tabular}

these experiments that the number of vegetative fronds and turions formed inside the idifferent rings is much smaller than in the controls. A greater number of vegetative fronds (Table 2) inside the rings was obtained in aerated cultures.

\section{Aeration of the nutrient solution}

It is commonly known that the aeration of the nutrient solutions has a positive effect on the vegetative growth. In order to examine the influence of this factor on the formation of turions, cultures were aerated every day at exactly the same time for 10 minutes during the first ten days and for 30 minutes in the following ones. In this way a daily supply of oxygen was assured to the deeper situated layers of the fronds and their roots. Culture vessels with 35 and $300 \mathrm{~cm}^{2}$ of upper free area of 
Table 3

Influence of aeration on the formation of turions

\begin{tabular}{c|c|c|c|c}
\hline $\begin{array}{c}\text { Area of the nut- } \\
\text { rient solution } \mathrm{cm}^{2}\end{array}$ & $\begin{array}{c}\text { Number of ve- } \\
\text { getative fronds }\end{array}$ & $\begin{array}{c}\text { Number of tu- } \\
\text { rions }\end{array}$ & $\begin{array}{c}\text { Ratio of vegetati- } \\
\text { ve fronds to turions }\end{array}$ & $\begin{array}{c}\text { Day of turion } \\
\text { formation }\end{array}$ \\
\hline $\begin{array}{c}35 \\
\text { (control) } \\
35\end{array}$ & 276 & 1112 & $1: 4.0$ & 23 \\
$\begin{array}{c}\text { (aerated) } \\
300 \\
\text { (control) } \\
300\end{array}$ & 1269 & 793 & $1: 1.5$ & 22 \\
$\left(\begin{array}{c}\text { (aerated) } \\
\text { (n) }\end{array}\right.$ & 1767 & 7243 & $1: 5.3$ & 34 \\
\hline
\end{tabular}

the nutrient solutions were used (corresponding to 100 and $750 \mathrm{ml}$ of the solution). The turions were counted after 10 weeks (Table 3 ).

The results indicate that many more vegetative fronds are formed when the nutrient solution is stirred in consequence of aeration, whereas the number of turions formed by one vegetative frond is much lower than in the controls.

\section{The volume of the nutrient solution}

It has been already stated that the formation of turions coincides with the moment when the upper surface of the culture solution is covered completely by vegetative fronds. It can not be, however, ruled out that this phenomenon is mere coincidence and the true factor res-

\section{Table 4}

Influence of the amount of nutrient solution on the turions formation

\begin{tabular}{c|c|c|c|c|c}
\hline $\begin{array}{c}\text { Amount of } \\
\text { nutrient } \\
\text { solution ml }\end{array}$ & $\begin{array}{c}\text { Number of } \\
\text { vegetative } \\
\text { fronds }\end{array}$ & $\begin{array}{c}\text { Number of } \\
\text { turions }\end{array}$ & $\begin{array}{c}\text { Ratio of vegeta- } \\
\text { tive fronds to } \\
\text { turions }\end{array}$ & $\begin{array}{c}\text { Day of } \\
\text { turion } \\
\text { formation }\end{array}$ & $\begin{array}{c}\text { \% of cove- } \\
\text { red area }\end{array}$ \\
\hline & & & & & \\
10 & 61 & 141 & $1: 2.3$ & 7 & 35 \\
25 & 144 & 461 & $1: 3.2$ & 12 & 78 \\
50 & 181 & 716 & $1: 3.9$ & 17 & 96 \\
75 & 216 & 969 & $1: 4.5$ & 21 & 100 \\
100 & 264 & 1038 & $1: 3.9$ & 22 & 100
\end{tabular}

ponsible for the formation of turions is the exhaustion of one or several mineral ions in the nutrient solution. In order to settle this question experiments were carried out in which the volume of the nutrient solutions was idifferent $(10,25,50,75$ and $100 \mathrm{ml})$ and their upper areas 
maintened on the same level (Table 4). The nutrient solutions were not aerated. Plants were cultured in a light thermostat for 7 weeks, after which time the turions were counted.

As it results from Table 4 the covering of the whole area of the nutrient solution by vegetative fronds is not an indispensible condition for the formation of turions because in a smaller volume turions are formed much earlier (most probably as a consequence of an exhaustion of the nutrient solution). The results of former experiments $(100 \mathrm{ml}$ nutrient solution) suggest that there is a correlation between the quantity of compounds in the nutrient solution and its upper area, which causes that turions are formed only when the whole surface of the nutrient solution is occupied by vegetative fronds. It is possible that at this moment a new factor is operating which limits further vegetative growth (most probably exhaustion of a compound in the nutrient solution). This factor, during further carbon assimilation leads to a storage of carbohydrates in turions. Concomitantly with the increase of the volume of the nutrient solution the number of vegetative fronds and turions increases as well.

5. The influence of the concentration of sucrose in the nutrient solution on the formation of turions

Sucrose was added to the nutrient solutions in amounts sufficient to obtain the following concentrations: $0,1,0,25,0,50,1,2$ and $5 \%$. The number of formed turions was counted after 7 weeks (with the exception of controls where turions were counted after 10 weeks because of a slow vegetative growth and delayed formation of turions).

\section{Table 5}

Formation of turions in dependence on the concentration of sucrose in the nutrient solution

\begin{tabular}{l|c|c|c|c|c}
\hline $\begin{array}{c}\text { Sucrose content } \\
\text { in nutrient } \\
\text { solution \% }\end{array}$ & $\begin{array}{c}\text { Number of } \\
\text { vegetative } \\
\text { fronds }\end{array}$ & $\begin{array}{c}\text { Number of } \\
\text { turions }\end{array}$ & $\begin{array}{c}\text { Ratio of vegetative } \\
\text { fronds to turions }\end{array}$ & $\begin{array}{c}\text { Day of turions } \\
\text { formation }\end{array}$ & $\begin{array}{c}\% \text { of covered } \\
\text { area }\end{array}$ \\
\hline & & & & & \\
0 & 76 & 106 & $1: 1.4$ & 33 & 80 \\
0,1 & 164 & 263 & $1: 1.6$ & 28 & 100 \\
0,25 & 206 & 389 & $1: 1.9$ & 25 & 100 \\
0.50 & 188 & 606 & $1: 3.2$ & 23 & 100 \\
1 & 194 & 766 & $1: 3.9$ & 20 & 100 \\
2 & 136 & 784 & $1: 5.8$ & 13 & 85 \\
5 & 28 & 69 & $1: 2.5$ & 8 & 8
\end{tabular}

It can be seen from Table 5 that the time required for the turions formation decreases with the increase of sucrose concentration. However, $5 \%$ sucrose cause already growth disturbances and leads to frond defor- 
mations. In the concentration range from 0,1 to $2 \%$ the number of turions formed by one vegetative frond increases with the sucrose concentration. The formation of turions is most delayed on nutrient solutions with no sucrose.

6. Extent of sucrose utilization during growth and the formation of turions

The addition of sucrose $(1 \%)$ to the nutrient solution stimulated the vegetative growth and the formation of turions. Bertrand's method was used for the determination of the amounts of sucrose utilized during

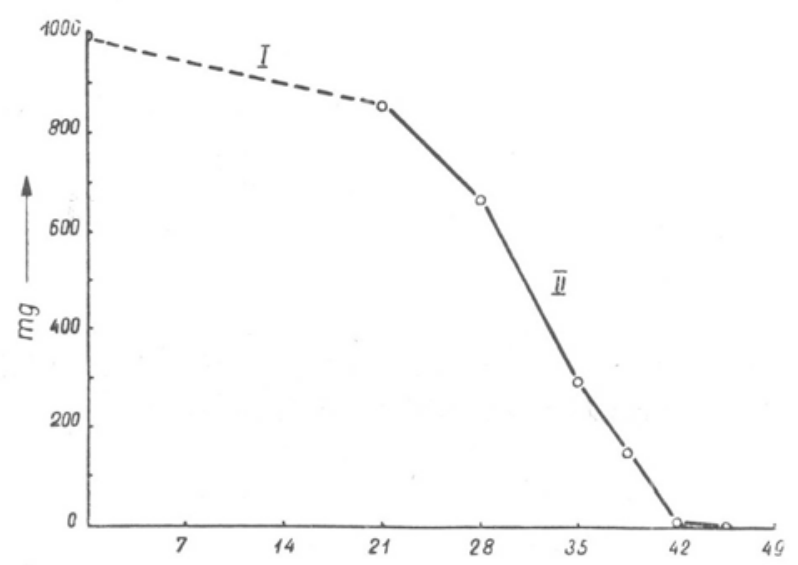

Fig. 2. Decrease of sucrose content in $100 \mathrm{ml}$ nutrient solution during the growth of vegetative fronds and turions formation. Abscissae - days, ordinates - sucrose content in $\mathrm{mg} / 100 \mathrm{ml}$ nutrient solution

$I$ - period of vegetative growth of fronds, $I I-$ period of turions formation

the formation of turions. The analyses were performed once a week from the moment the turions began to be formed (i.e. after 3 weeks of vegetative growth) till the end of this process. The analyses were carried out on 3 samples.

The analytical results indicate that there is still a considerable amount of sucrose $(860 \mathrm{mg}$ in $100 \mathrm{ml})$ in the nutrient solution at the beginning of turions formation, whereas, at the end of this process ( 7 weeks) a complete absence of sucrose in the nutrient solution is observed. Thus the most intensive utilization of sucrose coincide with the period of turions formation.

7. The formation of turions on old nutrient solutions

In order to determine whether the plant, respectively the turions secrete any substances inducing the formation of turions, an experiment utilising an old nutrient solution (in which turions had already once 
Table 6

Formation of turions in mixtures of old and fresh nutrient solutions with $1 \%$ sucrose

\begin{tabular}{|c|c|c|c|c|c|c|}
\hline \multicolumn{2}{|c|}{ Nutrient solution $\mathrm{ml}$} & \multirow{2}{*}{$\begin{array}{l}\text { Number of } \\
\text { vegetative } \\
\text { fronds }\end{array}$} & \multirow{2}{*}{$\begin{array}{l}\text { Number } \\
\text { of turions }\end{array}$} & \multirow{2}{*}{$\begin{array}{l}\text { Ratio of vege- } \\
\text { tative fronds } \\
\text { to turions }\end{array}$} & \multicolumn{2}{|c|}{$\begin{array}{l}\text { Beginning of turions } \\
\text { formation }\end{array}$} \\
\hline old & fresh & & & & day & $\begin{array}{c}\% \text { of covered } \\
\text { area }\end{array}$ \\
\hline 100 & - & 4 & 29 & $1: 7.5$ & 5 & 4 \\
\hline $75^{\prime}$ & 25 & 56 & 276 & $1: 5.0$ & 13 & 17 \\
\hline 50 & 50 & 73 & 457 & $1: 6.3$ & 14 & 25 \\
\hline 25 & 75 & 147 & 744 & $1: 5.1$ & 22 & 100 \\
\hline- & 100 & 273 & 954 & $1: 3.5$ & 23 & 100 \\
\hline
\end{tabular}

been formed) was carried out. An analysis of the nutrient solution made after the removal of all the rests of dead plants showed a complete absence of sucrose. Nutrient solutions were prepared by mixing in various proportions the old solution and a fresh one (Table 6). On the old nutrient solution supplemented with $1 \%$ sucrose but with no other mineral compounds, turions were formed already after 5 days (Table 6). Most probably the exhaustion of one or several mineral compounds in

\section{Table 7}

Formation of turions in mixtures of old and fresh nutrient solutions in dependence on of sucrose concentration

\begin{tabular}{|c|c|c|c|c|c|c|c|}
\hline \multicolumn{2}{|c|}{ Nutrient solution $\mathrm{ml}$} & \multirow{2}{*}{$\begin{array}{c}\text { Sucrose } \\
\text { content } \\
\%\end{array}$} & \multirow{2}{*}{$\begin{array}{l}\text { Number of } \\
\text { vegetative } \\
\text { fronds }\end{array}$} & \multirow{2}{*}{$\begin{array}{l}\text { Number } \\
\text { of turions }\end{array}$} & \multirow{2}{*}{$\begin{array}{l}\text { Ratio of vege- } \\
\text { tative fronds } \\
\text { to turions }\end{array}$} & \multicolumn{2}{|c|}{$\begin{array}{l}\text { Beginning of turions } \\
\text { formation }\end{array}$} \\
\hline old & fresh & & & & & day & $\begin{array}{c}\% \text { of covered } \\
\text { area }\end{array}$ \\
\hline 100 & - & 0 & 3 & 6 & $1: 2.0$ & 18 & 3 \\
\hline 75 & 25 & 0.25 & 77 & 239 & $1: 3.1$ & 22 & 80 \\
\hline 50 & 50 & 0.50 & 98 & 449 & $1: 4.6$ & 22 & 100 \\
\hline 25 & 75 & 0.75 & 120 & 502 & $1: 4.2$ & 24 & 100 \\
\hline 一 & 100 & 1.00 & 247 & 894 & $1: 3.6$ & 23 & 100 \\
\hline
\end{tabular}

the nutrient solution is the factor limiting the vegetative growth. A delay in the formation of turions is concomitant with the increase of the proportion of fresh nutrient solution. However, with no sucrose in the old nutrient solution the turions were formed after 18 days (Table 7). Their number, moreover, was small in comparison to that of turions. formed on an old nutrient solution of the same concentration but supplemented with $1 \%$ sucrose (Table 6 ). 
8. Prolongation of the vegetative stage of the fronds and the formation of turions

Experiments were performed in order to obtain more information on the connection between the formation of turions and the percent of the upper surface covered by fronds. At fixed dates single plants were transferred from a culture to fresh nutrient solutions containing $1 \%$ sucrose. The successive transfers were made when the percentages of the surface covered by frond amounted to $20 \%$ ( 7 days), $35 \%$ (10 days),

Table 8

Shifting of the period of turions formation by plants transferred to a fresh nutrient solution in dependence of the time of transfer. \% denote the percentages of the upper area of the nutrient solution occupied by vegetative fronds at the time of transfer. $t$ - number of turions, $v . f$. - number of vegetative fronds

\begin{tabular}{|c|c|c|c|c|c|c|}
\hline Day & Control & $\begin{array}{c}20 \% \text { area } \\
7 \text {-th day }\end{array}$ & $\begin{array}{l}35 \% \text { area } \\
10 \text {-th day }\end{array}$ & $\begin{array}{l}60 \% \text { area } \\
12 \text {-th day }\end{array}$ & $\begin{array}{l}90 \% \text { area } \\
14-\text { th day }\end{array}$ & $\begin{array}{c}100 \% \text { area } \\
16 \text {-th day }\end{array}$ \\
\hline 21 & 2 & 一 & - & - & - & - \\
\hline 22 & 10 & - & - & - & - & - \\
\hline 23 & 18 & 3 & - & - & - & - \\
\hline 24 & 27 & 9 & - & - & - & - \\
\hline 25 & 43 & 13 & - & - & - & - \\
\hline 26 & 74 & 16 & 一 & - & - & - \\
\hline 27 & 102 & 31 & - & - & - & - \\
\hline 28 & 115 & 45 & 1 & - & - & - \\
\hline 29 & 123 & 59 & 3 & - & - & - \\
\hline 30 & 130 & 70 & 7 & 2 & - & - \\
\hline 31 & 138 & 88 & 12 & 4 & - & 4 \\
\hline 32 & 145 & 109 & 35 & 16 & - & 9 \\
\hline 33 & 164 & 128 & 47 & $27^{\circ}$ & 4 & 24 \\
\hline 34 & 181 & 136 & 55 & 42 & 16 & 30 \\
\hline 35 & 199 & 140 & 68 & 47 & 25 & 43 \\
\hline 36 & 225 & 144 & 78 & 57 & 36 & 63 \\
\hline 37 & $\imath$ & 152 & 90 & 70 & 52 & 75 \\
\hline 38 & ? & 173 & 117 & 84 & 72 & 98 \\
\hline 39 & ? & 198 & 147 & 101 & 82 & 119 \\
\hline 40 & 2 & 214 & 161 & 121 & 94 & 142 \\
\hline ? & ? & $?$ & 2 & $\imath$ & 2 & ? \\
\hline 2 & t. 428 & 327 & 276 & 223 & 227 & 297 \\
\hline 49 & v.f. 148 & 163 & 170 & 158 & 147 & 134 \\
\hline Ratio of ${ }^{*}$ & $1: 3$ & $1: 2$ & $1: 1.6$ & $1: 1.4$ & $1: 1.5$ & $1: 2.2$ \\
\hline
\end{tabular}

* Ratio of the number of vegetative fronds to number of turions

$60 \%$ (12 days), $90 \%$ (14 days) and $100 \%$ (16 days). The time required for the turions formation and their number were recorded.

The experimental results indicate that the later the vegetative fronds were transferred to fresh solutions the later the turions were formed. 
On this basis the induction of turions formation in the first stage of vegetative growth must be ruled out, otherwise the fronds transferred latest to fresh solutions should rapidly form turions.

In the final phase of the vegetative growth the plant is to a certain extent predisposed for turions formation. The fact of enabling the plant to reverse to the vegetative growth (by transferring to a fresh nutrient solution) causes a retardation of turions formation which increases with the lenght of the optimal conditions maintained for vegetative growth before transfering the frcids to a fresh nutrient solution. And so, by giving the plant optimal ccniditions permanently, its normal vegetative developmental cycle may be indefinitely prolonged. And reverse, by making worse the developmental conditions (e.g. by isupplying a smaller dose of mineral ccmpounds), the vegetative growth may be retarded and the turions formed at an earlier time (Table 4).

\section{DISCUSSION}

The results of our experiments indicate that there are several factors inducing the turions formation. In naturai environment turions are formed from July to late autumn in conditions of high temperature and great light intensity ( $\mathrm{J} \mathrm{acobs}$ 1947). Laboratory investigations show ( $\mathrm{J}$ a cobs 1947) that turions may also be formed at $10^{\circ} \mathrm{C}$ and low light intensity (about $540 \mathrm{lux}$ ). A constant temperature about $30^{\circ} \mathrm{C}$ is optimal for the formation of turions provided that the intensities of other factors are also optimal or nearly optimal. J a co bs (1947) established that light is one of the most important factors inducing turions formation. He observed a considerable stimulation of formation of turions in fronds exposeid for 20 hours to light intensities above 1000 lux.

Addition of $\mathrm{CO}_{2}$ in otherwise favourable conditions may lead to a considerable preponderance of photosynthesis over respiration. This, in turn promotes the storage of carbohydrates in turions ( $\mathrm{J}$ a c o b s 1947). Nitrogen deficiency in the nutrient solution is another factor inducing the formation of turions, it inhibits the vegetative growth and in consequence also promotes the accumulation of starch in turions ( $\mathrm{J}$ a cobs 1947). H ens sen (1954) mentions that a high concentration of storage substances by inactivating the amylases influences indirectly the formation of turions.

Our investigations have shown that there are several factors influencing the formation of turions; the ratio of the upper free area of the nutrient solution to its volume is one of them. The smaller the area, the earlier it is covered with vegetative fronds, which form several layers and consequently the earlier the turions appear. It is assumed that in the upper layers the growth is inhibited by a deficiency of nutrient compounds, and in lower layers by a difficult access of oxygen. The 
absorption of mineral ions by the growing fronds and the resulting depletion of the nutrient solution is another factor influencing the formation of turions. Sucrose has a reverse effect because an increase of its concentration promotes the induction of turions formation. Only a small amount of sucrose is utilized for the vegetative growth, the greater part being used for turions formation. Aeration of the nutrient solution stimulates the vegetative growth but does not influence the formation of turions.

It can be seen, from the above given data, that there are numerous and diverse factors influencing the formation of turions. This fact makes difficult the elucidation of the process of turions formation. There is, however, one property common to all these factors, all they cause inhibition of vegetative growth. Thus, a hypothesis can be advanced that inhibition of vegetative growth is the factor directly responsible for the induction of turions formation, whereas, all the other mentioned factors influence this process only in an indirect way. The inhibition of vegetative growth, on the one hand, and an intensive metabolism on the other hand, are factors promoting the disposition for turions formation in the plant. This may be considered to be the result of an evolutional adaptation of the plant which enables the species to survive unfavourable life conditions. On the contrary by assuring optimal growth conditions (by periodically transferring the fronds to fresh nutrient solutions) an unlimited prolongation of vegetative development can be achieved.

\section{SUMMARY AND CONCLUSIONS}

The influence of various external factors on the turion formation of Spirodela polyrrhiza made the subject of special investigations. The experiments have shown that there are several external factors inducing the formation of turions in an indirect way.

1. Spirodela polyrrhiza was cultured in sterile cultures on nutrient solutions supplemented with $1 \%$ sucrose in constant light and temperature conditions.

2. Turions are formed much more rapidly in dense cultures on nutrient solutions presenting small free areas in comparison to their volumes.

3. Aeration of the nutrient solution accelerates the vegetative growth but does not influence the formation of turions.

4. Exhaustion of one (or more) mineral compounds in the nutrient solution induces the formation of turions.

5. A higher concentration of sucrose also induces the formation of turions and causes a concomitant weaker vegetative growth. The major part of sucrose is utilized for turion formation. 
A hypothesis, based on the obtained experimental results, has been advanced, that the inhibition of vegetative growth is the factor directly responsible for the induction of turion formation. Thus, every external factor which inhibit the vegetative growth induces in the plant a disposition for turion formation. On the contrary by providing optimal developmental conditions, the normal vegetative developmental cycle may be indefinitely prolonged.

I wish to express my most sincere thanks to prof. dr F. Górski, dr A. Z u$\mathrm{rzycka}$ and docent dr J. Zurzycki for their valuable advices in the course of this work.

Department of Plant Physiology

(Entered: 7.10.1962)

Polish Academy of Sciences

Kraków, Poland

\section{REFERENCES}

C zopek M. 1959 a. Cultivation of Polish Lemnaceae species in laboratory conditions. Acta Biol. Crac. Ser. Bot. II, 13-22.

$\mathrm{Czopek}$ M. $1959 \mathrm{~b}$. Researches on the physiology of formation and germination of turions in Spirodela polyrrhiza (L.) Schleiden. Acta Biol. Crac. Ser. Bot. II, 75-90.

C z o pek M. 1962. The oligodynamic action of light on the germination of turions of Spirodela polyrrhiza (L.) Schleiden. Acta Soc. Bot. Pol. 31, 703-722.

Hens sen A. 1954. Die Dauerorgane von Spirodela polyrrhiza (L.) Schleiden in physiologischer Betrachtung. Flora 141, 523-566.

H i $11 \mathrm{~m}$ a n W. S. 1961. The Lemnaceae, or duckweeds. A review of descriptive and experimental literature. The Bot. Rev. 27, 222-287.

J a c o bs D. L. 1947. An ecological life history of Spirodela polyrrhiza (greater duckweed) with emphasis on the turion phase. Ecol. Monographs 17, 437-469.

Kandeler R. 1955. Über die Blütenbildung bei Lemna gibba L. I. Kulturbedingungen und Tageslängenabhängigkeit. Zeitschr. f. Bot. 43, 61-71.

Landolt E. 1957. Physiologische und ökologische Untersuchungen an Lemnaceen. Ber. Schweiz. Bot. Ges. 67, 271-410. 